\title{
LA SUBESTIMACIÓN DE LA MORTALIDAD INFANTIL EN MÉXICO
}

\author{
EDUARDO CORDERO \\ El Colegio de México
}

\section{INTRODUCCIÓN}

Son muchos los factores de diverso origen asociados con la mortalidad y la morbilidad de los individuos en el primer año de vida; entre otros, la nutrición, las condiciones de la vivienda, los ingresos, la existencia y el uso adecuado de los servicios médicos. La deficiencia de estos factores determina la frecuencia de las defunciones tempranas. Estos factores se han clasificado en endógenos y exógenos, según su origen. Los primeros se refieren a la falta de viabilidad intrínseca para sobrevivir que proceden de la constitución del niño, sujeta a malformaciones eventuales y a las condiciones en que se desarrollan tanto el embarazo como el parto. Los factores exógenos abarcan todos aquellos de procedencia extrínseca como las infecciones, las intoxicaciones alimenticias y los accidentes.

La mortalidad infantil ${ }^{1}$ es un indicador sensible a cualquier cambio en ia estructura económicosocial, en particular la mortalidad exógena condicionada a los factores más fácilmente controlables. Los factores endógenos pueden manifestar mejoría en las condiciones económicas y sociales, dado que en general se encuentran muy estrechamente ligados entre sí, aunque su sensibilidad para reflejar de inmediato los cambios es menor.

Los países llamados desarrollados acusan una mortalidad infantil reducida; en cambio, aquellos que presentan bajos ingresos por habitantes y agudos problemas sociales se caracterizan por una elevada proporción de defunciones durante el primer año de la existencia. Por ello, la tasa de mortalidad infantil puede considerarse como buen indicador de la situación social de una determinada comunidad; tanto es así que la Secretaría de las Naciones Unidas recomienda su inclusión dentro de los indicadores demográficos utilizados para medir el nivel de vida alcanzado por los países. ${ }^{2}$

1 Mortalidad infantil se refiere específicamente a defunciones de niños menores de un año. La tasa de mortalidad infantil, que equivale a una probabilidad de muerte $\left(q_{o}\right)$, indica defunciones por cada mil nacidos vivos.

2 Naciones Unidas, Report on International Definitions and Measurements of Standards and Levels of Living. Nueva York, 1954. 
Sin embargo, las entidades con poco desarrollo económico y social, incluidas entre las que mayor necesidad tienen de contar con un fiel indicador de mortalidad infantil, presentan deficiencias en la información básica, y en consecuencia los índices obtenidos con ella adolecen de serias desviaciones de difícil medición.

Como se verá en el curso de este trabajo, el caso de México es un ejemplo fehaciente de esta realidad. Si bien se considera que sus estadísticas de defunciones registran un poco más del $90 \%$ de las muertes totales acaecidas, mínimo requerido por los acuerdos internacionales para estimarlas "completas", 3 es posible que las defunciones omitidas no se distribuyan uniformemente entre todas las edades, sino que se concentren en las de menores de un año. En caso de que las omisiones en este nivel sean importantes y exista además un posible subregistro de los nacimientos, la tasa de mortalidad infantil resulta evidentemente subestimada.

Para subsanar las deficiencias de los registros de nacimientos y defunciones en el primer año de vida, se elaboran en este trabajo estimaciones de los niveles probables de la tasa de mortalidad infantil en México, dada la importancia del indicador como instrumento de análisis y para la planeación de la salud pública.

La pauta del estudio la proporcionó el análisis de la evolución de la mortalidad por edades en México, donde se comprobó una falta de correspondencia entre las tasas de mortalidad infantil y otros indicadores de la mortalidad por edades de la tabla de vida, según lo observado por otras experiencias, lo que hizo ver la necesidad de nuevas estimaciones de la mortalidad en el primer año de vida. ${ }^{4}$

Se parte del hecho, investigado con anterioridad, ${ }^{5}$ de que las probabilidades de muerte por edades se correlacionan entre sí, es decir, que las tasas de una edad determinada y aquellas que las anteceden o preceden pueden ajustarse a una línea de regresión de primer, segundo o tercer grado. En esta forma se han elaborado métodos para estimar los niveles de la mortalidad general a partir de indicadores particulares, tales como la tasa de mortalidad infantil cuando se carece de la información apropiada y confiable para conocer la esperanza de vida al nacimiento. ${ }^{6}$ La metodología adoptada en este documento parte de idénticos principios, aunque en forma inversa: se estiman las tasas de mortalidad infantil con base en el grupo precedente (uno a 4 años), cuya relación en México no es la observada en las tablas de mortalidad de países con estadísticas confiables o en las tablas modelo de Naciones Unidas, deducidas de las primeras. ${ }^{7}$

Se ha considerado conveniente dividir el trabajo en cuatro partes.

3 Naciones Unidas, Manual de métodos de estadisticas vitales. Estudios Metodológicos. Serie F, № 7. Nueva York, 1955.

4 Eduardo Cordero, Análisis de la evolución de la mortalidad en México, 19301960. Trabajo de investigación para obtener la Maestría en Demografía en El Colegio de México, 1967 (inédito).

5 Naciones Unidas, Boletín de Población de las Naciones Unidas, Núm. 6. 1962, p. 156.

6 K. R. Gabriel e Ilana Ronen, "Estimates of Mortality from Infant Mortality Rates", Population Studies. Londres, Vol. XII, Núm. 2. 1958.

7 Naciones Unidas, Modelos de mortalidad por sexo y edad. Tablas modelo de mortalidad para países insuficientemente desarrollados. ST/SOA/Ser. A/22. 1960. 
La primera consiste en una revisión de la evolución de la tasa de mortalidad infantil, calculada con base en los registros anuales de defunciones de menores de un año y de nacimientos, de 1896 a 1965.

En la segunda etapa, con base en las tablas de mortalidad, se comprueba la hipótesis de la subestimación de las tasas de mortalidad infantil a través de su asociación con otros indicadores de mortalidad: la esperanza de vida al nacimiento, la probabilidad de muerte de mujeres de 5 a 34 años y la mortalidad neonatal.

En la tercera parte se estiman las tasas de mortalidad infantil probables a nivel nacional en los años 1930, 1940, 1950, 1960 y 1965, así como en cada entidad federativa durante los años 1950, 1960 y 1965. En esta parte se analizan los resultados obtenidos, buscando asociar los niveles de subestimación de las tasas de mortalidad infantil con las características de urbanización, alfabetismo y fecundidad.

Por último, se efectúa un breve análisis de la evolución de la mortalidad infantil por medio de las tasas corregidas y se hacen algunos comentarios pertinentes.

\section{LA MORTALIDAD INFANTIL EN MÉXICO DE 1896 A 1965}

La evolución de la mortalidad infantil en el presente siglo ha sido notable, en especial en los países poco desarrollados. En América La-

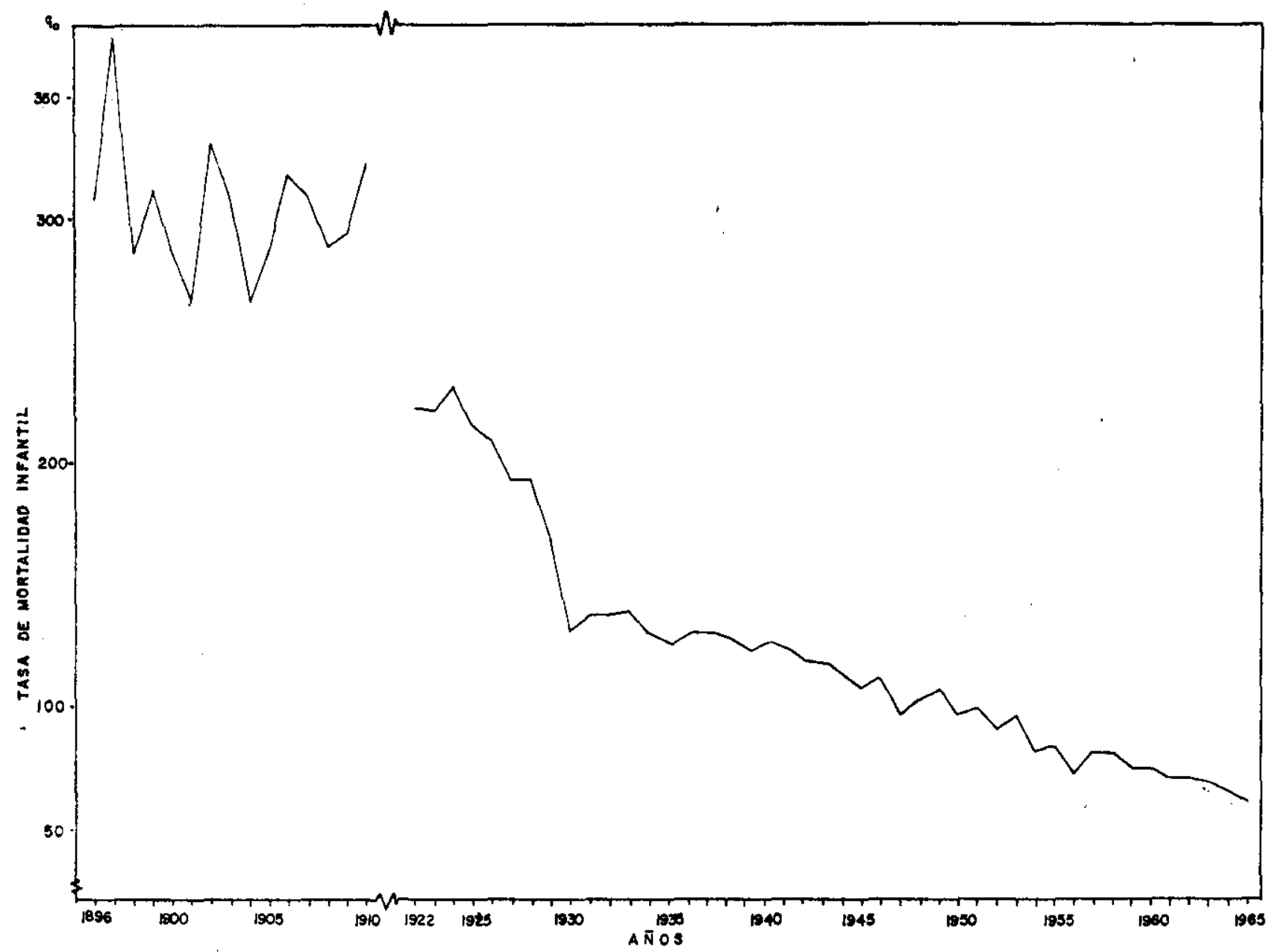

Fuente: Cuadro 1.

Gráfica 1 
tina se ha logrado reducir la tasa de mortalidad infantil en un plazo relativamente corto, si se le compara con el transcurrido en los países en que se inició el descenso de la mortalidad en los siglos XVIII y XIX. En México, según datos oficiales, la tasa ha disminuido en pocos años a niveles que países como Suecia requirieron mucho más de un siglo para lograr, por la ausencia de los avances médicos y sanitarios con que se cuenta en la actualidad.

Como se observa en el cuadro 1 y la gráfica 1, entre 1896 y 1910 la tasa de mortalidad infantil se muestra con altos niveles y notables oscilaciones entre un mínimo de 266.4 (1901) y un máximo de 376.7 (1897). En esta época los registros vitales deben haber sido más irregulares que en cualquier otro período posterior, exceptuando naturalmente el revolucionario de los años 1911 a 1921, cuando no existen registros en absoluto. Para disminuir el efecto de estas oscilaciones de las tasas anuales, se utilizan los promedios aritméticos de tres años en la cuantificación de los cambios sufridos por las tasas de mortalidad infantil.

\section{Cuadro 1}

MÉXICO: TASAS DE MORTALIDAD INFANTIL, 1896-1965

\begin{tabular}{|c|c|c|c|c|c|}
\hline$A \bar{n} 0$ & Tasa & Año & Tasa & 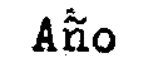 & Tasa \\
\hline $\begin{array}{l}1896 \\
1897 \\
1898 \\
1899 \\
1900 \\
1901 \\
1902 \\
1903 \\
1904 \\
1905 \\
1906 \\
1907 \\
1908 \\
1909 \\
1910 \\
1911-21 \\
1922 \\
1923 \\
1924 \\
1925\end{array}$ & $\begin{array}{l}309.0 \\
376.7 \\
286.9 \\
312.5 \\
286.8 \\
266.4 \\
331.9 \\
310.1 \\
266.8 \\
286.7 \\
318.5 \\
310.6 \\
288.1 \\
294.3 \\
323.1 \\
-2-. \\
223.1 \\
222.4 \\
232.2 \\
215.9\end{array}$ & $\begin{array}{l}1926 \\
1927 \\
1928 \\
1929 \\
1930 \\
1931 \\
1932 \\
1933 \\
1934 \\
1935 \\
1936 \\
1937 \\
1938 \\
1939 \\
1940 \\
1941 \\
1942 \\
1943 \\
1944 \\
1945\end{array}$ & $\begin{array}{l}209.4 \\
193.0 \\
193.4 \\
167.6 \\
131.6 \\
137.7 \\
137.5 \\
139.3 \\
130.3 \\
125.7 \\
130.8 \\
130.8 \\
128.0 \\
122.6 \\
125.7 \\
123.0 \\
118.2 \\
117.2 \\
113.5 \\
107.9\end{array}$ & $\begin{array}{l}1946 \\
1947 \\
1948 \\
1949 \\
1950 \\
1951 \\
1952 \\
1953 \\
1954 \\
1935 \\
1956 \\
1957 \\
1958 \\
1959 \\
1960 \\
1961 \\
1962 \\
1963 \\
1964 \\
1965 \\
1966\end{array}$ & $\begin{array}{r}110.6 \\
96.4 \\
101.7 \\
106.4 \\
96.2 \\
98.8 \\
89.8 \\
95.2 \\
80.5 \\
83.3 \\
71.0 \\
80.1 \\
80.1 \\
74.4 \\
74.2 \\
70.2 \\
69.9 \\
68.5 \\
64.5 \\
60.7 \\
62.9\end{array}$ \\
\hline
\end{tabular}

Fuente: Secretaría de Industria y Comercio, Dirección General de Estadística, Anuarios estadísticos de los Estados Unidos Mexicanos, 1958-1959, 1964 y 19641965. 
De 1897 a 1909 se localiza una disminución del $6.9 \%$, la que puede deberse, por una parte, a la mejoría del nivel de vida como efecto de los inicios de la industrialización, aunque sólo benefició a una mínima parte de la población. ${ }^{8}$ Por otra parte, es posible que esa disminución sea tan sólo aparente, debido a la mencionada irregularidad en los registros de defunciones y nacimientos.

Entre 1909 y 1923 se registra uno de los cambios más significativos en la tasa de mortalidad infantil. Las cifras acusan una disminución del $25.1 \%$ al descender de 301.8 a 225.9 defunciones de menores de un año por mil nacidos vivos. En este renglón cabe establecer nuevamente la duda sobre la fidelidad de los registros en la época prerrevolucionaria, plagada aún de situaciones adversas que coadyuvan a aumentar la mortalidad y el subregistro. Todavía en los años subsecuentes se observan irregularidades considerables, entre 1923 y 1930, cuando según la información se experimenta la baja más notable del período estudiado, $35.5 \%$, la que es debida, más que a ganancias en la mortalidad, a un aumento en el registro de nacimientos por las disposiciones legales de 1929 .

En los años siguieñtes, la tasa de mortalidad infantil continúa descendiendo, con decrementos del $15.0 \%$ entre 1930 y $1940,18.8$ y $27.5 \%$ en los dos decenios siguientes y 14.1 entre 1960 y 1965 . En esta última fecha, la tasa de mortalidad infantil representa sólo el $19.3 \%$ de la observada en promedio en 1897 y el $43 \%$ de la de 1930.

La tasa de mortalidad infantil en México en 1965 (60.7) es baja en comparación con la de los países con más alta mortalidad en el mundo: en Malí se estiman 250.0, en el Congo 200.0 y en Haití 176.9 defunciones de menores de un año por cada mil nacimientos. ${ }^{9}$ Pero a pesar de su disminución es aún muy elevada cuando se compara con Suecia, Países Bajos, Noruega, Finlandia, Australia y Nueva Zelandia, donde las tasas son inferiores a 20.0.10 Puede apreciarse cuán amplio es el margen que resta para reducir ese componente de la mortalidad en México.

Las tasas de mortalidad infantil de Hungría en el período 1956-1958 y Argentina en 1960, ${ }^{11}$ presentan niveles parecidos a los de México en 1965 (60.7). Dado que la tasa de mortalidad infantil es un fiel indicador de la situación social, deberían esperarse niveles de vida parecidos en los tres países en las épocas correspondientes, así como condiciones sociales superiores a las de Yugoslavia, que en 1963 acusaba una tasa de 84.2,12 Profundizando un poco en el análisis por medio de otros indicadores económicosociales, tales como el ingreso per capita, la población rural, el alfabetismo y el número de habitantes por médico, ${ }^{13}$ se llega necesariamente a pensar en una seria deficiencia de la tasa de mortalidad infantil de México debida principalmente a la omi-

8 Leopoldo Solís M., "Hacia un análisis general a largo plazo del desarrollo económico de México", Demograffa Y Economf́, Vol. I, Núm. 1, 1967.

9 Naciones Unidas, Demographic Yearbook, 1965.

10 Ibid.

11 Ibid.

$12 \cdot$ Ibid.

13 Naciones Unidas, Demographic Yearbook y World Population Data Sheet; OEA, América en cifras. 
sión de defunciones en el registro, sin descartar la posibilidad de que existan diferencias ocasionadas por otras razones.

En general, la mayoría de los registros de los sucesos vitales en los primeros años de vida resultan incompletos, lo cual obliga a establecer dudas sobre los índices basados en ellos. El problema principal se refiere a los niños que fallecen a poco de nacer y que no se registran ni como nacimientos ni como defunciones. A esto puede añadirse que muchas veces no se sabe con certeza que es lo que se considera un "nacimiento vivo". Existe aún desconcierto entre las opiniones más destacadas de los médicos en lo relativo a la separación de las defunciones infantiles, las muertes fetales y los abortos. ${ }^{14}$ Por ello, las deficiencias en el registro de las defunciones se concentran por lo general en la infancia y más concretamente en las primeras horas o días de la vida, lo que dificulta la medición y el análisis de la mortalidad infantil.

\section{Evaluación dE LAS TASAS DE MORTALIDAd INFANTIL}

Como las tasas de mortalidad infantil calculadas con los registros anuales de defunciones de menores de un año y los nacimientos tienen el defecto de mezclar generaciones distintas, de aquí en adelante se hablará de las probabilidades de morir a la edad cero de las tablas de vida, que han sido calculadas en forma más refinada. ${ }^{15}$

Para evaluar las tasas de mortalidad infantil obtenidas a través de las tablas de vida existentes para 1930,1940, 1950 y 1960,16 se calculó el valor de la asociación entre dichas tasas (correlación simple) y la esperanza de vida al nacimiento $\left(e_{0}^{o}\right)$; se estableció la relación entre la probabilidad de muerte a la edad cero $\left(q_{0}\right)$ y la probabilidad de muerte de mujeres de 5 a 34 años $\left({ }_{29} q_{5}\right)$; y se hicieron comparaciones entre la mortalidad neonatal y la infantil total.

El empleo de los métodos indicados es el que en gran medida ha llevado a considerar, por una parte, la importancia de la subestimación de las tasas de mortalidad infantil y, por otra, a buscar la implementación para efectuar nuevas estimaciones.

a) Para establecer el grado de asociación de la esperanza de vida al nacer y la tasa de mortalidad infantil, se calculó el índice de correlación simple, que resultó ser de -0.966 (véase el cuadro 10), es decir, que a una elevada mortalidad infantil corresponde un bajo nivel de la esperanza de vida al nacimiento. Por otra parte, se tomaron en cuenta las tablas modelo de Naciones Unidas ${ }^{17}$ y la línea de regresión calculada por Gabriel y Ronen,18 quienes, además, evalúan la confiabilidad del sistema de Naciones Unidas, obteniendo una desviación promedio para 150 tablas de +2.133 , con una varianza del estimado

14 United Nations. Foetal, Infant and Early Childhood Mortality, Vol. 1, The Statistics. Nueva York, 1954, pp. 3-9.

15 Diagrama de Lexis. Véase Roland Pressat, El análisis demográfico. México, Fondo de Cultura Económica, 1967.

I6 Raúl Benítez Zenteno y Gustavo Cabrera Acevedo, Tablas abreviadas de mortalidad de la población de México, 1930, 1940, 1950 y 1960. México, El Colegio de México, 1968.

17 Naciones Unidas, Modelos..., op. cit.

I8 Gabriel y Ronen, op. cit. 
de 18.68 con 147 grados de libertad, lo que indica que el cálculo de Naciones Unidas tiende a sobrestimar la esperanza de vida.

Sin embargo, a pesar de este inconveniente del sistema, se consideró adecuado para efectuar la primera evaluación de las tasas de mortalidad infantil. En esta forma se calcularon las esperanzas de vida al nacimiento que les corresponderían a cada probabilidad de morir a la edad cero de las cuatro tablas de mortalidad.

Los resultados de estos cálculos pueden observarse en el cuadro 2 . La esperanza de vida al nacer que le correspondería a la tasa de mortalidad de 1930 debería ser 8.44 años mayor que la observada, 7.83 años mayor en 1940, 3.88 en 1950 y es hasta 1960 cuando se obtiene un valor aproximado de sólo 0.02 años mayor que la observada.

\section{Cuadro 2}

MÉxico: Cálculo de la esPeranza DE VIDA al Nacimiento SEGÚN LAS TASAS DE MORTALIDAD INFANTIL OBSERVADAS, 1930-1960 •

\begin{tabular}{cccc}
\hline Fecha & $\begin{array}{c}{ }^{o e_{o}}{ }^{\mathrm{a}} \\
\text { Observada } \\
(1)\end{array}$ & $\begin{array}{c}{ }^{o} e_{o} \mathrm{~b} \\
\text { Calculada } \\
\text { (2) }\end{array}$ & $\begin{array}{c}\text { Diferencia } \\
(2)-(1)\end{array}$ \\
\hline 1930 & 36.86 & 45.30 & 8.44 \\
1940 & 41.45 & 49.28 & 7.83 \\
1950 & 49.69 & 53.57 & 3.88 \\
1960 & 58.93 & 58.95 & 0.02 \\
\hline
\end{tabular}

a Benítez Z. y Cabrera, Tablas... op. cit.

b Calculada con la línea de regresión:

$o e_{o}=75.230-238.08 q_{o}+239.46\left(q_{0}\right)^{2}$

\section{Cuadro 3}

México: Cálculo de las tasas de MORTAlidad INFantil SEgúN LOS NIVELES DE LA ESPERANZA DE VIDA OBSERVADA AL NACIMIENTO, $1930-1960$

\begin{tabular}{cccc}
\hline Fecha & $\begin{array}{c}q_{o}{ }^{a} \\
\text { Observada } \\
(1)\end{array}$ & $\begin{array}{c}q_{o}{ }^{b} \\
\text { Calculada } \\
(2)\end{array}$ & $\begin{array}{c}\text { Diferencias } \% \\
(2)-(1)\end{array}$ \\
\hline 1930 & 147.63 & $\frac{12)}{100}$ \\
1940 & 124.61 & 209.06 & 29.38 \\
1950 & 101.29 & 171.11 & 27.18 \\
1960 & 73.87 & 122.11 & 17.05 \\
\hline
\end{tabular}

a Benítez Z. y Cabrera, Tablas... op. cit.

b Calculada con la línea de regresión:

$$
q_{o}=\frac{238.08+\sqrt{(238.08)^{2}-4(239.46)\left(75.230-o e_{0}\right)}}{2(239.46)}
$$


A la inversa, se obtuvieron las tasas de mortalidad infantil a partir de la esperanza de vida al nacimiento observada correspondiente. La tasa de mortalidad infantil resulta para 1930 menor en $29.38 \%$. En las dos siguientes fechas las diferencias continúan siendo importantes, aunque se reducen en 1960 a $0.01 \%$, diferencia que prácticamente no es significativa (cuadro 3 ).

A juzgar por estos resultados, no es sino hasta 1960 cuando dejan de darse diferencias entre las tasas de mortalidad infantil estimadas por medio de la esperanza de vida al nacimiento a través de la línea de regresión calculada con la información de 150 tablas de mortalidad y las tasas de mortalidad infantil obtenidas directamente con las estadísticas vitales de México. Debe tenerse en cuenta que el sistema es bastante heterodoxo y subestima la mortalidad, de donde se puede esperar que aun en 1960 exista subestimación de la tasa de mortalidad infantil, como se verá a continuación.

b) La probabilidad de muerte de mujeres de 5 a 34 años es recomendada por Naciones Unidas como buen indicador de la mortalidad general. ${ }^{19}$ La base es proporcionada por los resultados del análisis factorial de la mortalidad. ${ }^{20}$

El estudio de la mortalidad a través del análisis factorial lo iniciaron Ledermann y Breas. ${ }^{21}$ Estos investigadores logran identificar tres componentes principales independientes y Naciones Unidas continúa el estudio identificando dos más.

La componente fundamental nunca se separa mucho de la tabla tipo de mortalidad correspondiente a la misma esperanza de vida al nacer, o sea que representa la mortalidad natural biológica correspondiente a una situación determinada. Todas las variaciones observadas son debidas a los factores que se adicionan para formar la segunda, tercera, cuarta y quinta componentes.

La segunda y tercera componentes actúan en ambos sentidos sobre la mortalidad, es decir, que pueden aumentarla o disminuirla, y especialmente actúan sobre las personas de edad adulta y sobre los niños a partir de un año. Sobre estos últimos sus efectos son en sentido opuesto al de los adultos y ancianos.

La cuarta y quinta componentes son las que, junto con la fundamental, interesan para la evaluación de las tasas de mortalidad infantil, ya que esta se encuentra afectada, según el análisis, sólo por la componente fundamental y el conjunto de factores que constituyen la cuarta componente, teniendo esta última poco o ningún efecto sobre la mortalidad en las edades 5 a 34 años.

Sin embargo, la quinta componente, que se refiere a la masculinidad de la mortalidad, es decir, la diferencia de la mortalidad por sexo, no acusa comportamiento muy claro en la mortalidad de personas de 5 a 34 años, por lo que fue elegida la probabilidad de mujeres de esas edades.

En el cuadro 4 se incluyen datos sobre mortalidad infantil y de

19 Naciones Unidas, Boletín..., op. cit., p. 66.

20 Ibid., p. 196.

21 Sully Lederman y Jean Breas, "Les dimensions de la mortalité". Population, París, 14: año, Núm. 4, 1956, pp. 653-659. 


\section{Cuadro 4}

INDICES DE MORTALIDAD INFANTIL DE VARIOS PAÍSES RELACIONADOS CON ÍNDICES DE MORTALIDAD DE MUJERES DE 5 A 34 AÑOS

$\begin{array}{ccccc}P \text { aí } & \text { A } \tilde{n} 0 & q_{0} .1000 & 29^{q_{5.1000}} & (4) \div(3) .100 \\ \text { (1) } & (2) & (3) & \text { (4) }\end{array}$

\begin{tabular}{llrrr} 
Argentina a/ & $1946-48$ & 71.35 & 67.638 & 94.8 \\
Chiloa/ & $1960-61$ & 117.01 & 63.610 & 54.4 \\
Chilea/ & $1952-53$ & 120.26 & 89.409 & 74.3 \\
Puerto Ricoa/ & 1960 & 37.75 & 31.130 & 82.5 \\
$\begin{array}{l}\text { Panamá (zona del } \\
\text { Canal)/ }\end{array}$ & $1959-61$ & 48.35 & 23.145 & 47.9 \\
Méxicob/ & 1930 & $147.63^{\circ}$ & 258.897 & 175.4 \\
Méxicob/ & $1939-41$ & 124.61 & 201.745 & 261.9 \\
Méxicob/ & $1949-51$ & 101.29 & 127.839 & 126.2 \\
xéxicob/ & $1959-61$ & 73.87 & 79.257 & 107.3 \\
\hline
\end{tabular}

a Naciones Unidas, Demographic Yearbook, 1950, 1955 y 1963.

b R. Benítez y G. Cabrera, op. cit.

mujeres de 5 a 34 años, así como el porcentaje que representan estos últimos respecto de los primeros en varios países de América Latina con buenas estadísticas. En este cuadro puede observarse que ese porciento varía entre el 94.8 en Argentina y el 47.9 en la zona del Canal de Panamá; en cambio en México resulta mayor del $100 \%$ en las cuatro fechas.

Esta relación estudiada en las tablas modelo,22 resulta mayor del $100 \%$ para niveles bajos de esperanza de vida al nacimiento y sólo es menor cuando el valor de este último indicador alcanza los 55 años. En el nivel más bajo de estas tablas, con una esperanza de vida al nacer de 20 años, la probabilidad de muerte de mujeres de 5 a 34 años representa el $141.9 \%$ de la tasa de mortalidad infantil, o sea que nunca llega ese porciento a representar los valores que alcanza México en las tablas de 1930 y 1940 . En las dos tablas siguientes, según los niveles de la esperanza de vida al nacimiento, resultan aún elevadas las proporciones de la mortalidad de mujeres respecto de la mortalidad infantil.

c) Especialmente en el primer año de vida se observan, cuando disminuye la mortalidad, las variaciones ocasionadas por dos tipos de causas de muerte: las defunciones debidas a factores endógenos

22 Naciones Unidas, Métodos para preparar proyecciones de población por sexo y edad. ST/SOA/Ser.A/25. 1956. 
o mortalidad neonatal,23 característica de las primeras semanas de vida, y la mortalidad exógena o posnatal debida fundamentalmente a factores del medio ambiente.

Dichas variaciones en esta edad operan en la siguiente forma: a medida que disminuye la mortalidad ocasionada por factores externos, la proporción de muertes neonatales es mayor, ya que la disminución de este componente de la mortalidad infantil es mucho más lenta y por otra parte los límites de la reducción en este caso son menores.

Así, en Suecia, el país que cuenta con la mortalidad infantil más baja del mundo (17 defunciones de menores de un año por cada mil nacidos vivos), la mortalidad neonatal representa el $75.9 \%$ de las defunciones infantiles totales. Canadá, con una mortalidad infantil intermedia (30.0 en 1960 ) registra un $63.7 \%$ de defunciones neonatales. Esta importancia de la mortalidad neonatal ha crecido en el tiempo, cuando la mortalidad infantil ha disminuido; en 1930 representaba el $56.5 \%$ en Suecia y el $48.3 \%$ en Canadá (cuadro 5 ).

Chile, con una mortalidad infantil notablemente mayor que la de México, tiene porcientos más elevados de muertes neonatales, excepto en 1960 cuando en México aumenta y en Chile disminuye en forma irregular. Es posible deducir de esto que la mortalidad neonatal debería tener en México una importancia mayor que la de Chile, durante los años 1930, 1940 y 1950 . En 1960 el porciento de muertes neonatales debería ser sólo un poco menor que el que representa Canadá en 1930, ya que la tasa de mortalidad infantil de este país fue de 79.6 y la de México en 1960 es de 73.9.

En México, la tasa de mortalidad infantil descendió de 147.6 en 1930 a 124.6 en $1940,101.3$ en 1950 y 73.9 en 1960. Paralelamente, el

\section{Cuadro 5}

PoRCiENTO DE LA MORTALIDAD NEONATAL CON RESPECTO a LA INFANTIL tOTAL
A $\tilde{\mathrm{n}} \circ \mathrm{g}$
Héxico a/
Chile b/
Canadá b/
Suecia $\underline{b} /$

\begin{tabular}{lllll}
\hline 1930 & 34.6 & 41.9 & 48.3 & 56.5 \\
1940 & 33.9 & 43.3 & 52.9 & 61.3 \\
1950 & 34.3 & 42.2 & 57.4 & 69.5 \\
1960 & 38.3 & 31.5 & 63.7 & 75.9 \\
\hline
\end{tabular}

a R. Benítez y G. Cabrera, Tablas... op. cit.

b Para Chile, Canadá y Suecia la fuente es: Juan C. Elizaga, Apuntes de mortalidad, CELADE, mimeografiado.

23 Mortalidad neonatal es aquella que se refiere a defunciones de niños menores de 28 días o un mes. La mortalidad posnatal abarca defunciones de niños de uno a 11 meses. 
número absoluto de las defunciones varió poco: 107921 en 1930, 110137 en 1940,113032 en 1950 y 119316 en 1960. Sin embargo, y no obstante la disminución considerable de la mortalidad infantil, de 1930 a 1950 la proporción de muertes neonatales fue aproximadamente la misma, lo que posiblemente refleja más los defectos del registro de las defunciones que los cambios en la estructura de la mortalidad infantil, que deberían ser pronunciados. En 1960 la proporción de muertes neonatales aumentó significativamente, de tal forma que a la vez que es posible hablar de una mejoría en el registro, se pueden observar los cambios que deben operar en la mortalidad neonatal respecto de la infantil total.

Lo anterior muestra claramente que gran parte de las omisiones en el registro de defunciones de menores de un año deben ser neonatales y particularmente aquellas omitidas en el curso de la primera semana de vida de los recién nacidos. A su vez, esto explica en parte la posible omisión simultánea en los registros de nacimientos y defunciones.

En resumen, las tres pruebas realizadas para comprobar la subestimación de las tasas de mortalidad infantil de México coinciden en sus resultados: a la esperanza de vida al nacimiento observada corresponderían tasas de mortalidad infantil más elevadas que las obtenidas con la información existente, aun en 1960 cuando el sistema indica una correspondencia entre ambos indicadores. Las tasas de mortalidad infantil tienen niveles bajos si se las compara con las probalidades de muerte de mujeres de 5 a 34 años, según el ejemplo dado por países con estadísticas confiables. Por último, la mortalidad neonatal no tiene la importancia relativa que se observa en otras experiencias. Estas dos últimas pruebas revelan que existe subestimación aun en 1960, coincidiendo con el método que se utiliza en la siguiente sección para estimar las tasas de mortalidad infantil probables.

\section{ESTIMACIÓN DE LAS TASAS DE MORTALIDAD INFANTIL PROBABLES}

1. Estimaciones a nivel nacional. Los resultados anteriores llevan directamente a la estimación de nuevas tasas de mortalidad infantil; en consecuencia, el método empleado puede considerarse como un cuarto sistema para evaluarlas.

En primer término, se tomaron en cuenta las tablas modelo de Naciones Unidas, donde se calculó la probabilidad de morir entre cero y cuatro años $\left({ }_{4} q_{0}\right)$ a partir de la tasa de mortalidad infantil $\left(q_{0}\right)$ y en forma derivada se estimaron las probabilidades de morir entre uno y cuatro años $\left({ }_{4} q_{1}\right)$. Sin embargo, el ajuste de estas últimas a una parábola de segundo o tercer grado mostró desviaciones acusadas al tratar de reconstruir las $q_{0}$, en especial en los niveles extremos. ${ }^{24}$

Por esta razón, principalmente, no se emplearon dichas tablas para estimar las tasas de mortalidad infantil de México. En su lugar se utilizaron tablas de 27 países con buenas estadísticas para calcular el índice de correlación entre las tasas de mortalidad infantil y las probabilidades de morir entre uno y cuatro años $(0.986$, véase el cua-

24 Naciones Unidas, Modelos..., op. cit. 
dro 10), y la línea de regresión correspondiente por mínimos cuadrados. Estos resultados fueron muy semejantes a los obtenidos por Gabriel y Ronen, empleados finalmente en este trabajo para estimar las tasas probables de mortalidad infantil por considerarse más adecuados, dado que el número de casos observados es considerablemente mayor ( 150 tablas de mortalidad). A su vez, este método tiene deficiencias debidas a la no uniformidad o posibles deficiencias de la información básica en los niveles altos de mortalidad, o sea que en mayor o menor grado, algunos países presentan problemas de información similares a los de México. ${ }^{25}$

En el cuadro 6, columna 3, se especifican las tasas de mortalidad infantil estimadas para todo el país, así como los niveles de subestimación en los años de 1930, 1940, 1950, 1960 y 1965. Estas estimaciones se efectuaron a través de la línea de regresión:

$$
q_{0}=\frac{4^{\mathrm{q}} 1-0.024233}{0.8027} .26
$$

La subestimación de las tasas de mortalidad infantil se calcula en cerca del $40 \%$ durante los años de 1930 y 1940 y en $35 \%$ en 1950 ; en cambio en 1960 esa subestimación se ha reducido al $16.3 \%$ y en 1965 al $15.7 \%$.

2. Estimación de las tasas de mortalidad infantil de cada entidad federativa. Los resultados a nivel nacional proporcionan tan sólo una

Cuadro 6

México: Cálculo de las $\mathrm{q}_{0}$, total de la República

\begin{tabular}{lcccc}
\hline Fecha & $\begin{array}{c}4_{1}{ }_{1} 1000 \\
a /\end{array}$ & $\begin{array}{c}q_{0} .1000 \\
\text { Observados } \\
(2)\end{array}$ & $\begin{array}{c}q_{0} .1000 \\
\text { Calculados } \\
(1)\end{array}$ & $\begin{array}{c}\text { Omisión } \\
(3)-(2)\end{array}$ \\
\hline 1930 & 171.84 & 147.63 & 244.27 & 39.6 \\
1940 & 146.16 & 124.61 & 207.29 & 39.9 \\
1950 & 100.60 & 101.29 & 155.52 & 34.9 \\
1960 & 46.58 & 73.87 & 88.22 & 16.3 \\
1965 & 38.06 & 65.42 & 77.60 & 15.7 \\
\hline
\end{tabular}

a De 1930 a 1960 las probabilidades de muerte se obtuvieron de las tablas de mortalidad para México de Benítez y Cabrera, op. cit. Para 1965 se calcularon previamente a través del diagrama de Lexis con datos proporcionados por la Dirección General de Estadística, sobre nacimientos y defunciones de 1959 a 1966.

26 Gabriel y Ronen, op. cit. 


\section{Cuadro 7}

MÉXico: Cálculo dE LAS TASAS de MORTALIdAd INFANTIL, 1950 a

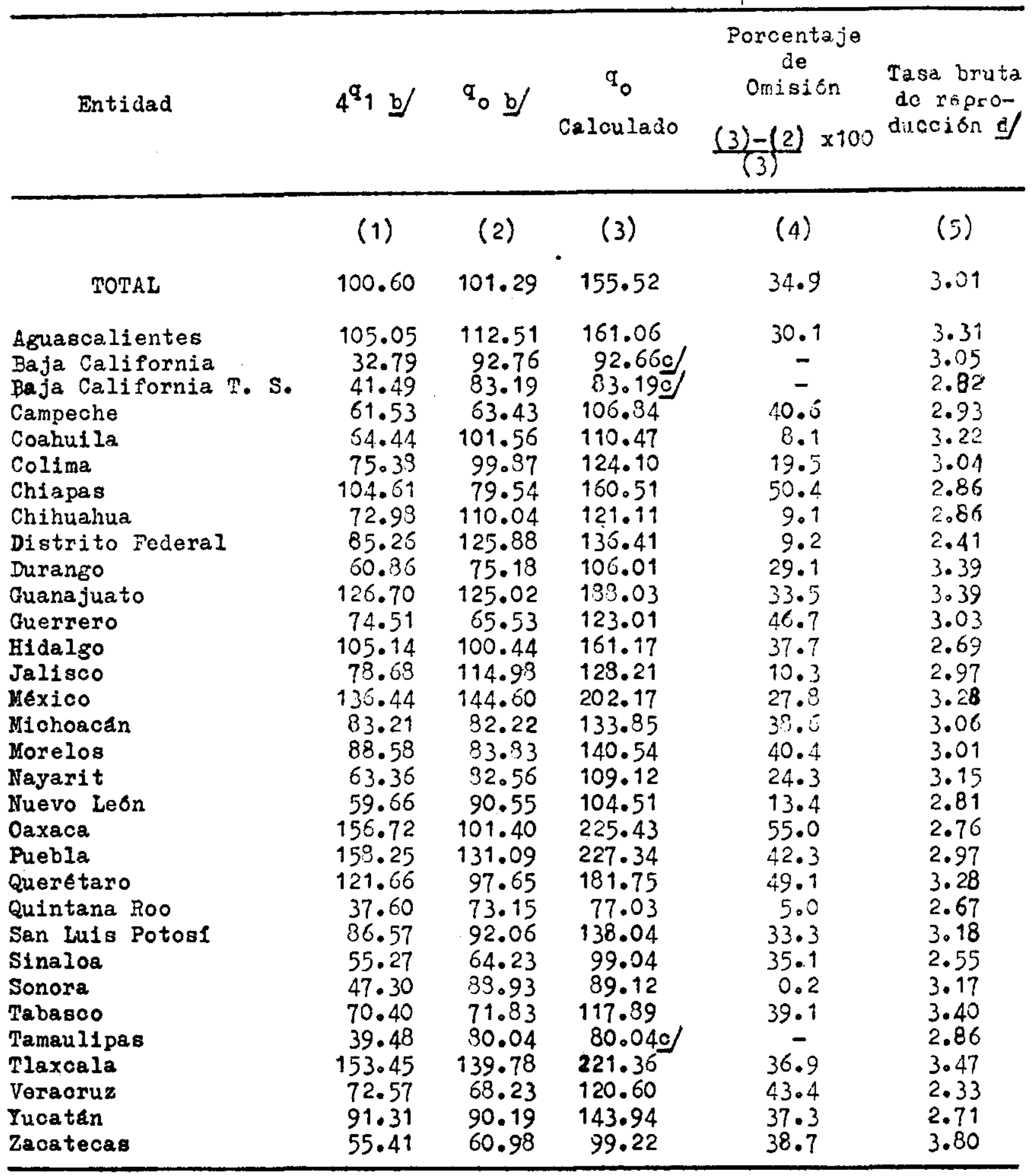

a Calculadas con la línea da regresión: $q_{0}=\frac{41+0.024233}{0.8027}$

b Probabilidades obtenidas de las tablas de mortalidad por estados, elaboradas por Raúl Benítez Z. y Gustavo Cabrera A., Centro de Estudios Económicos y Demográficos, El Colegio de México. Inéditas.

c Les correspondería una tasa menor según el nivel de la $4^{\mathrm{q}} 1$.

d Calculadas con los datos sobre nacimientos por edad de las madres para 1951 proporcionados por la Dirección General de Estadística. 
Cuadro 8

México: Cálculo de las tasas de mortalidad infantil, 1960

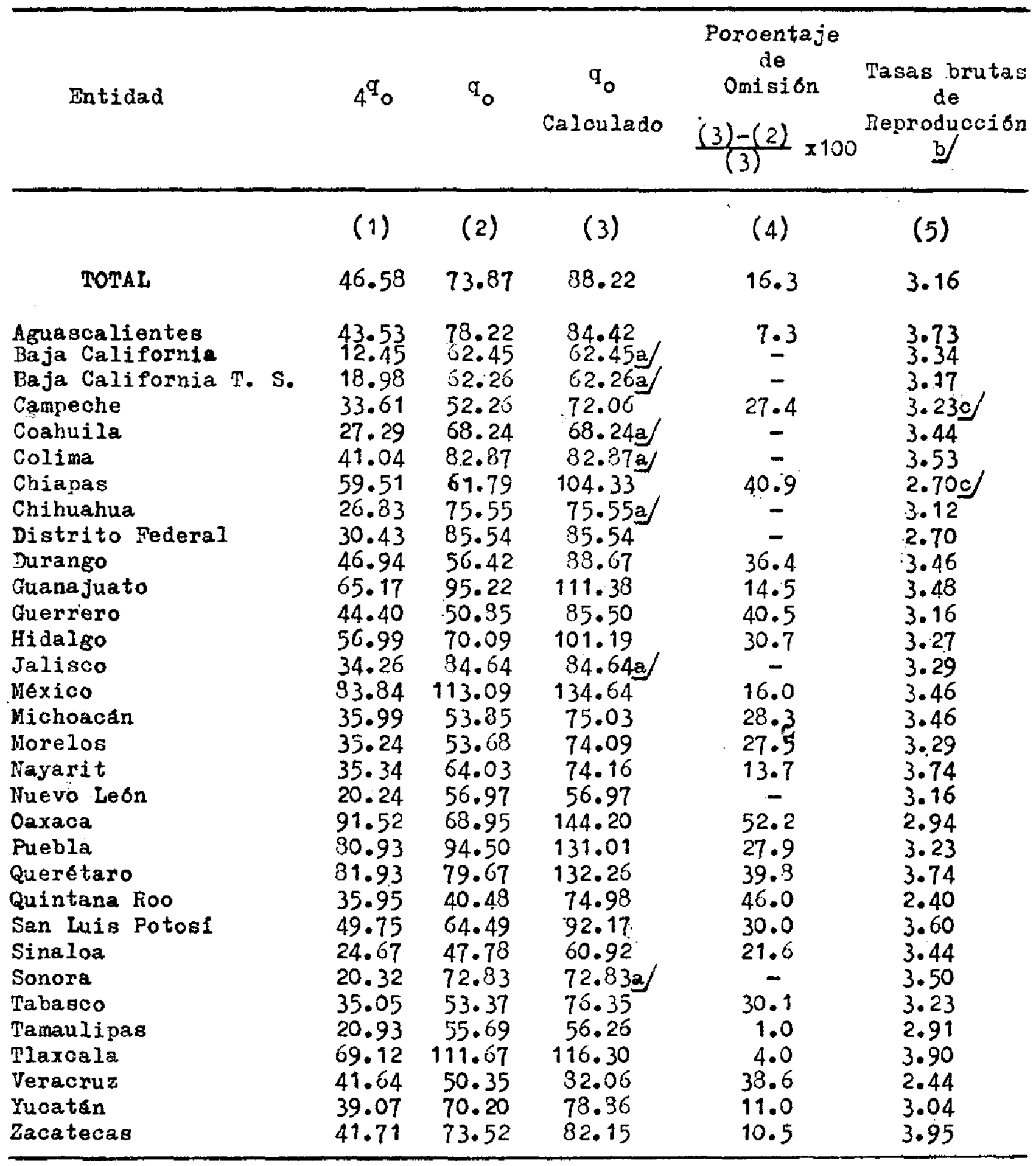

Fuente: Las mismas del cuadro 3-A.

a Les correspondería una tasa menor según el nivel de las $4^{\mathrm{q}} 1$.

b R. Benítez y G. Cabrera, Proyecciones de la población de México, 1960-1980. Banco de México, 1966.

c Corregidas de R. Benítez y G. Cabrera. Proyecciones... op. cit. 
base para conocer las proporciones de la anomalía de las estadísticas de México, por lo que resulta necesario contar con una estimación del grado de omisión cuando menos a nivel de entidad federativa.

Para efectuar este cálculo, se han utilizado las tablas de mortalidad por estados de 1950 y 1960.27 Las tasas requeridas de 1965 fueron cal-

\section{Cuadro 9}

MÉXico: Cálculo de las tasas de MoRTalidad INFantil, 1965

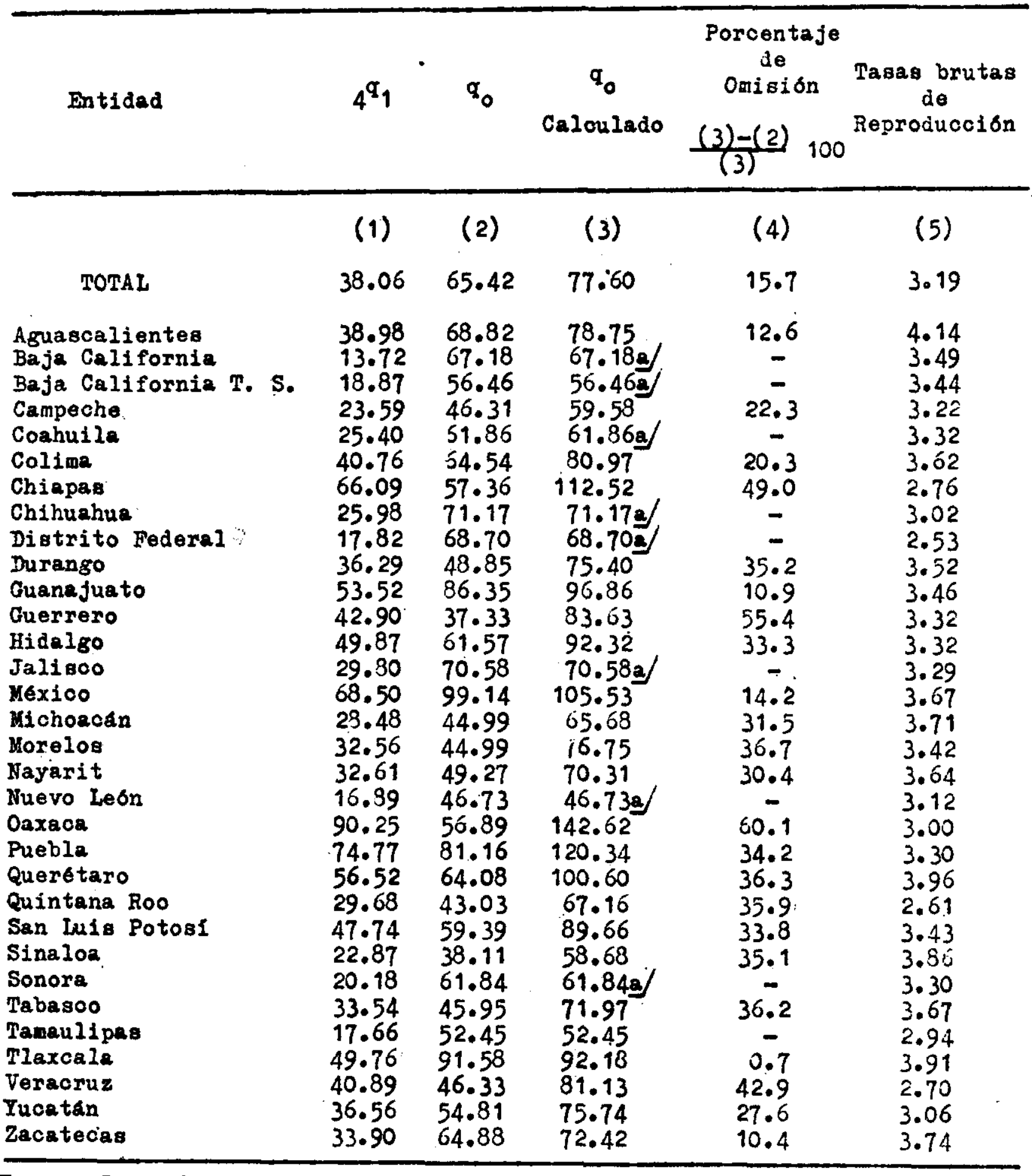

Fuente: Las mismas del cuadro 3-A.

a Les corresponde una tasa menor que la observada, según la relación con la $4^{\mathrm{a}} 1$.

27 Raúl Benítez Z. y Gustavo Cabrera A., Tablas abreviadas de mortalidad de México, por estados, 1949-1951 y 1959-1961. Centro de Estudios Económicos y Demográficos, El Colegio de México. Inéditas. 
culadas previamente para la elaboración de este trabajo. Los resultados se aprecian en los cuadros 7,8 y 9 , junto con las probabilidades de muerte de niños de uno a 4 años y las tasas de mortalidad infantil observadas, las que proporcionan la base para calcular el nivel de la subestimación.

El porciento de subestimación de la tasa resulta mayor en 1965 que en 1960 respecto a 15 de las 32 entidades federativas y mayor en 1960 que en 1950 en una (Quintana Roo). Esto puede explicarse teniendo en cuenta que las probabilidades de muerte de uno a 4 años registran decrementos menores que las tasas de mortalidad infantil registradas. Cabe hacer notar, además, que estas entidades son las que cuentan con mayores problemas en los registros vitales.

Las tasas estimadas se observan sistemáticamente descendentes en todos los estados durante las tres fechas estudiadas, con dos excepciones: Baja California y Chiapas, las cuales presentan en 1965 tasas de mortalidad infantil mayores que en 1960. Esto se debe a que las probabilidades de muerte de niños de uno a 4 años son menores en 1960 que en 1965, lo que puede deberse en ambos casos a una mejoría en el registro de defunciones de esas edades.

A este respecto, como es de esperar, los mayores porcientos de subestimación de las tasas de mortalidad infantil se dan principalmente en aquellas regiones del país habitadas por población rural de bajos niveles educacionales. Para comprobar esta afirmación, se ha calculado el grado de asociación de los niveles de subestimación de las tasas de mortalidad infantil en 1960 con el porciento de población alfabeta proporcionado por el censo de población de 1960, así como el grado de urbanización, definido como el porciento de la población que reside en núcleos urbanos, según el censo. El índice de correlación entre el grado de subestimación de la tasa de mortalidad infantil y el alfabetismo es de -0.766 , lo que indica que a mayor proporción de personas que saben leer y escribir corresponde menor grado de subestimación de la tasa. La urbanización y el grado de omisión presentan un índice de correlación de -0.755 , o sea que a las entidades con menor proporción de población radicada en centros urbanos de

Cuadro 10

INDICE DE CORRELACIÓN ENTRE DIFERENTES CONCEPTOS

Conceptos

Indice

Mortalidad infantil y esperanza de vida ${ }^{\text {a }}$

$-0.966$

Mortalidad de niños de 1 a 4 años y esperanza de vida ${ }^{a}$

Mortalidad infantil y mortalidad de niños de 1 a 4 años ${ }^{a}$

Omisión de defunciones de menores y alfabetismo ${ }^{b}$

Omisión de defunciones de menores y urbanización $b$

a Datos de 27 países y tablas modelo de Naciones Unidas.

b Datos referidos al Censo de Población de 1960 de México. 
2500 habitantes o más, corresponde mayor nivel de omisión en los registros vitales de menores.

Al relacionar el grado de omisión con los niveles de fecundidad, medida a través de las tasas brutas de reproducción de los años 1951, 1960 y 1965,28 (columna 4 de los cuadros 7, 8 y 9), se observa que los estados con alto porciento de subestimación presentan niveles relativamente bajos de fecundidad. En 1950 pueden mencionarse: Chiapas, Campeche, Guerrero, Michoacán, Morelos, Oaxaca, Puebla, Sinaloa, Veracruz y Yucatán. En 1960 y 1965 son notables los casos de Chiapas, Guerrero, Oaxaca, Quintana Roo y Veracruz. Al mejorar los registros, parece haber un incremento en la fecundidad; todas estas entidades, y algunas más, acusan en 1965 una tasa bruta de reproducción más elevada.

Este argumento parece fortalecer la hipótesis sobre la simultaneidad de la omisión en los registros de defunciones de menores de un

\section{Cuadro 11}

MÉxico: Estimación DE LOS REgISTrós taRdíos DE NACIMIENTos a

\begin{tabular}{|c|c|c|c|c|c|c|c|}
\hline \multirow{2}{*}{ Ã̃o } & \multicolumn{4}{|c|}{ Elad en la. Fecha del Regiotro } & \multicolumn{3}{|c|}{ Registros Tardios } \\
\hline & De 0 a 30 & $\begin{array}{l}\text { De } 31 \text { a } 364 \\
\text { d1as }\end{array}$ & $\%$ b/ & $\begin{array}{l}\text { De un año } \\
\text { omss }\end{array}$ & of & Total & $\begin{array}{l}\text { Por cada } 100 \\
\text { Feolintentos }\end{array}$ \\
\hline $\begin{array}{l}1951 \\
1952 \\
1953 \\
1954 \\
1955 \\
1956 \\
1957 \\
1958 \\
1959 \\
1900 \\
1961 \\
1962 \\
1963 \\
1904 \\
1965\end{array}$ & $\begin{array}{ll}28 & 721 \\
28 & 623 \\
30 & 542 \\
31 & 421 \\
31 & 914 \\
31 & 953 \\
32 & 994 \\
30 & 942 \\
33 & 849 \\
33 & 728 \\
33 & 918 \\
33 & 736 \\
33 & 371 \\
34 & 092 \\
33 & 956\end{array}$ & $\begin{array}{ll}240 & 779 \\
245 & 939 \\
240 & 103 \\
274 & 791 \\
284 & 989 \\
298 & 869 \\
321 & 601 \\
329 & 660 \\
353 & 206 \\
373 & 407 \\
389 & 623 \\
406 & 711 \\
423 & 717 \\
455 & 660 \\
459 & 762\end{array}$ & $\begin{array}{l}22.8 \\
23.0 \\
21.4 \\
22.9 \\
23.0 \\
23.2 \\
23.9 \\
24.9 \\
24.7 \\
25.3 \\
25.7 \\
25.8 \\
26.0 \\
26.5 \\
26.9\end{array}$ & $\begin{array}{rr}71 & 998 \\
76 & 190 \\
103 & 977 \\
102 & 552 \\
110 & 336 \\
133 & 936 \\
124 & 936 \\
120 & 116 \\
141 & 913 \\
135 & 238 \\
139 & 716 \\
171 & 132 \\
199 & 115 \\
216 & 157 \\
232 & 039\end{array}$ & $\begin{array}{l}6.1 \\
6.4 \\
8.6 \\
7.7 \\
3.0 \\
9.4 \\
3.4 \\
3.3 \\
3.9 \\
8.4 \\
8.2 \\
10.0 \\
11.3 \\
11.7 \\
12.3\end{array}$ & $\begin{array}{ll}341 & 498 \\
350 & 742 \\
379 & 622 \\
408 & 764 \\
427 & 239 \\
464 & 758 \\
479 & 531 \\
480 & 718 \\
533 & 973 \\
542 & 373 \\
563 & 257 \\
611 & 579 \\
656 & 197 \\
705 & 909 \\
735 & 757\end{array}$ & $\begin{array}{l}28.8 \\
29.3 \\
30.1 \\
30.5 \\
31.0 \\
32.6 \\
32.3 \\
35.2 \\
33.6 \\
33.7 \\
34.2 \\
35.9 \\
37.4 \\
39.2 \\
39.0\end{array}$ \\
\hline
\end{tabular}

Fuente: Secretaría de Industria y Comercio, Dirección General de Estadística, Anuarios estadisticos, 1955 a 1965.

a Los registros tardíos se estimaron bajo la hipótesis de que los registros de niños de todas las edades se efectuaron con una distribución uniforme. Así, cada día del mes hubo registros de niños de 1 a 30 días de edad; por ello, la probabilidad de que un niño de esas edades haya nacido el año anterior disminuye en 1/30 cada día del primer mes, lo que arroja un $51.6 \%$ de registros tardíos. En el caso de los niños de 31 a 364 días de nacidos, se calcula que el $54.17 \%$ son nacidos el año anterior. El total de las personas de uno y más años de edad provienen evidentemente de nacimientos de años anteriores al registro.

b Porciento de registros tardíos de niños de 0 a 364 días.

c Porciento de personas registradas de un año y más de edad, respecto del total de registros.

28 La tasa bruta de reproducción se define como la suma de todas las tasas por edad de fecundidad, tomando en cuenta sólo los nacimientos femeninos y considerando únicamente la población del sexo femenino. Puede igualmente definirse una tasa bruta de reproducción masculina. 
año y los nacimientos, de donde se desprende la necesidad de investigar la influencia de ambas omisiones en la tasa de mortalidad infantil.

Cabe agregar que el registro de nacimientos es también irregular por causa de los registros tardíos. En el cuadro 11 se estima el nivel de registros tardíos en un período de 15 años. Estos parecen aumentar con el tiempo, y aunque generalmente se supone que se compensan año tras año y su influencia es mínima, resulta necesario investigar sus efectos. Pero no se encuentra disponible la información requerida, tal como la desagregación de los grupos "registro de niños de 31 a 364 días de edad" y "registro de personas de un año y más".

\section{CONSIDERACIONES FinaleS}

La evolución de la mortalidad infantil en el total del país entre 1950 y 1965, ya corregida su tasa, que es mayor que la indicada por las estadísticas, registra no obstante un avance más optimista que aquel que se observa con las tasas oficiales. Estas últimas presentan una disminución del $36.9 \%$ en el período mencionado; en cambio con las tasas estimadas se obtiene un abatimiento del $50.1 \%$. El descenso mayor se encuentra entre 1950 y 1960 , cuando la tasa de mortalidad infantil pasa de 155.5 a 88.2 defunciones de menores de un año por 1000 nacimientos.

En cuanto a las entidades, el descenso ha sido más rápido en aquellas que contaban con una mortalidad infantil más elevada en 1950, tales como: Aguascalientes, Chiapas, Guanajuato, Estado de México, Morelos, Oaxaca, Puebla, Querétaro, Tlaxcala y Yucatán, aunque en todas ellas la mortalidad continúa siendo la más elevada del país.

Sería de interés estimar las tasas de mortalidad infantil por sexo, pero el método resulta impracticable, dado que en México existe una sobremortalidad femenina en las edades uno a 4 años y al aplicarlo por sexo arrojaría tasas de mortalidad infantil mayores para el sexo femenino, lo que resulta empíricamente inaceptable.

La sobremortalidad masculina en el primer año de vida es un fenómeno mundialmente observado, que parece obedecer fundamentalmente a causas biológicas. En cambio, la sobremortalidad femenina en las edades de uno a 4 años se presenta con mucha frecuencia, especialmente en las poblaciones con alta mortalidad. Por ejemplo, se encuentra en las tablas modelo de Naciones Unidas, hasta el nivel 45, cuando la esperanza de vida al nacimiento es de 42.5 años; de ahí en adelante hay una inversión y la mortalidad masculina es mayor. En Chile se presenta en las tablas de vida correspondientes a los años $1920,1930,1940,29$ y $1952 ; 30$ en 1960 se da la inversión y hay sobremortalidad masculina. ${ }^{31}$ En las tablas para Argentina de 1946-1948, 32 y

29 O. Cabello, J. Vildósola y M. Latorre, "Tablas de vida para Chile, 1920, 1930 y 1940", Revista Chilena de Higiene y Medicina Preventiva, Vol. VII, Núm. 3, septiembre de 1946 y Vol. IX, Núm. 2, junio de 1947.

30 Odette Tacla y José M. Pujol, Tablas abreviadas de mortalidad para Chile, 1952-1963 y 1960-1961. CELADE, Serie C, Núm. 11, Santiago de Chile.

31 Ibid.

32 Zulma Camisa, Tabla de mortalidad para la República Argentina, 1946-1948. CELADE, Santiago de Chile. 
1959-1961 33 también se presenta la sobremortalidad femenina en las edades de uno a 4 años, a pesar de contar con alta esperanza de vida. Por lo tanto, no puede considerarse una desviación de las estadísticas de México el hecho de que desde 1930 a 1965 se presente esta relación de la mortalidad entre los sexos.

Por último, debe hacerse patente que este intento de estimar las tasas de mortalidad infantil de México obedece principalmente a la necesidad de contar con buenos indicadores para conocer la realidad social del país, así como de subrayar la gran utilidad que tendría efectuar estudios directos en la población sobre los acontecimientos vitales. Pueden llevarse a cabo por medio de los censos de población o bien mediante investigaciones especiales para recabar esta información que los registros no captan en toda su magnitud.

33 Zulma Camisa, Tabta de mortalidad para la República Argentina, 1959-1961. CELADE, Santiago de Chile. 